\title{
Performance Analysis of Content Discovery for Ad-hoc Tactile Networks
}

\author{
Junaid Arshad ${ }^{\mathrm{a}}$, Muhammad Ajmal Azad ${ }^{\mathrm{b}}$, Khaled Salahc, Razi Iqbal ${ }^{\mathrm{d}}$, Muhammad Imran Tariq ${ }^{\mathrm{e}}$, Tariq Umer ${ }^{\mathrm{f}}$ \\ ${ }^{a}$ School of Computing and Engineering, University of West London, United Kingdom \\ ${ }^{b}$ Department of Computer Science, The University of Warwick, United Kingdom \\ ${ }^{c}$ Department of Electrical and Computer Engineering, Khalifa University, UAE \\ ${ }^{d}$ Department of Computer Information Technology, American University in The Emirates, UAE \\ e Japan Advanced Institute of Science and Technology,Ishikawa, Japan \\ ${ }^{f}$ Department of Computer Science, COMSATS Institute of IT, Pakistan
}

\begin{abstract}
Tactile Internet evolves communications to encompass sensory information such as smell and haptic sensations combining ultra-low latency with extremely high availability, reliability, and security. Tactile Internet is realized through underpinning technologies such as Multi-access Edge and Fog computing which facilitate decentralized infrastructures and machine to machine (M2M) communications. Mobile ad hoc networks (MANETs) form the foundation layer of such infrastructures, enabling direct communication between autonomous and decentralized devices such as sensors and vehicles. Among other applications, autonomous ad hoc vehicular networks (VANETs) and vehicle to vehicle (V2V) communications require efficient content discovery and quality of data transfer. The mobility patterns of vehicles within this communication model could effect the quality of data exchanged between devices in a tactile network. Several mobility models exist describing mobility patterns of mobile users in MANETs. In this paper, we present a first performance study to evaluate the impact of different mobility models on content discovery techniques for tactile Internet comprising of fast-moving vehicles and devices. This study combines direct and derived mobility metrics evaluating impact on content discovery and content dissemination using NS-3. Our simulation results indicate that unstructured techniques may not scale well within a tactile network of fast moving vehicles while maintaining low latency and could suffer from performance degradation in a saturated environment. Furthermore, simulation results also demonstrate the resilience of the unstructured content discovery protocol in mobility scenarios with proactive routing and diverse behavior.
\end{abstract}

Keywords: Tactile Internet, Mobility Models, Content Discovery, MANETs.

\section{Introduction}

Tactile Internet is referred to as a network of the Internet that enables high efficiency, ultra-low latency and maximum availability to facilitate Human to Machine (H2M) communication [1]. Example applications of tactile Internet range from robotics to traffic, from education to sports and from healthcare to smart grids [2, 3]. For instance, although tele-surgical operations have been experimented with since 2000, limitations in robotics and underlying technologies have inhibited its widespread adoption. Tactile Internet envisages overhauling tele-srugery by enabling a physician to instruct a telerobot at the patient's location, allowing remote physical examination with full $\mathrm{AV}$ and haptic feedback [3]. In order to achieve this, a defining characteristic of tactile Internet applications is the requirement to achieve a maximum cycle time of $1 \mathrm{~ms}$ $[1,4,5]$. This is essentially based on the expected reaction time of a human while interacting with systems of various types. For instance, human reaction to sudden, unforeseen incident requiring a muscular reaction is approximately 1 second whereas for a visual such as TV is 10 milliseconds [4]. Therefore, an affordable delay in H2M interactions such as moving a mouse pointer over the screen or responding to a Virtual Reality (VR) device is 1 millisecond. Achieving this delay will consequently enable Tactile Internet applications such as those allowing steering and control of real and virtual objects as identified by $[5,6]$.

In view of these characteristics of Tactile Internet, the advancements in communication technologies such as $4 \mathrm{G}$, $5 \mathrm{G}$ and millimeter wave $[6,7,8]$ are intuitive and fundamental to their achievement. Consequently, a key design principle for Tactile Internet is the use of local services and components which are close enough to end users so as to minimize delays. Within this context, Mobile Edge computing (recently evolved into Multi-access Edge Computing) (MEC) [9] and Fog Computing [10] represent paradigms enabling decentralized infrastructures to facilitate on-demand, flexible provision of localized services. More specifically, MEC and Fog computing are focused at enabling benefits of cloud computing closer to the user equipment such as radio network controller or macro base station for MEC and routers, switches or industrial controllers in the case of Fog computing thereby leveraging context, agility, and speed [1].

However, a node within a fog network is expected to 


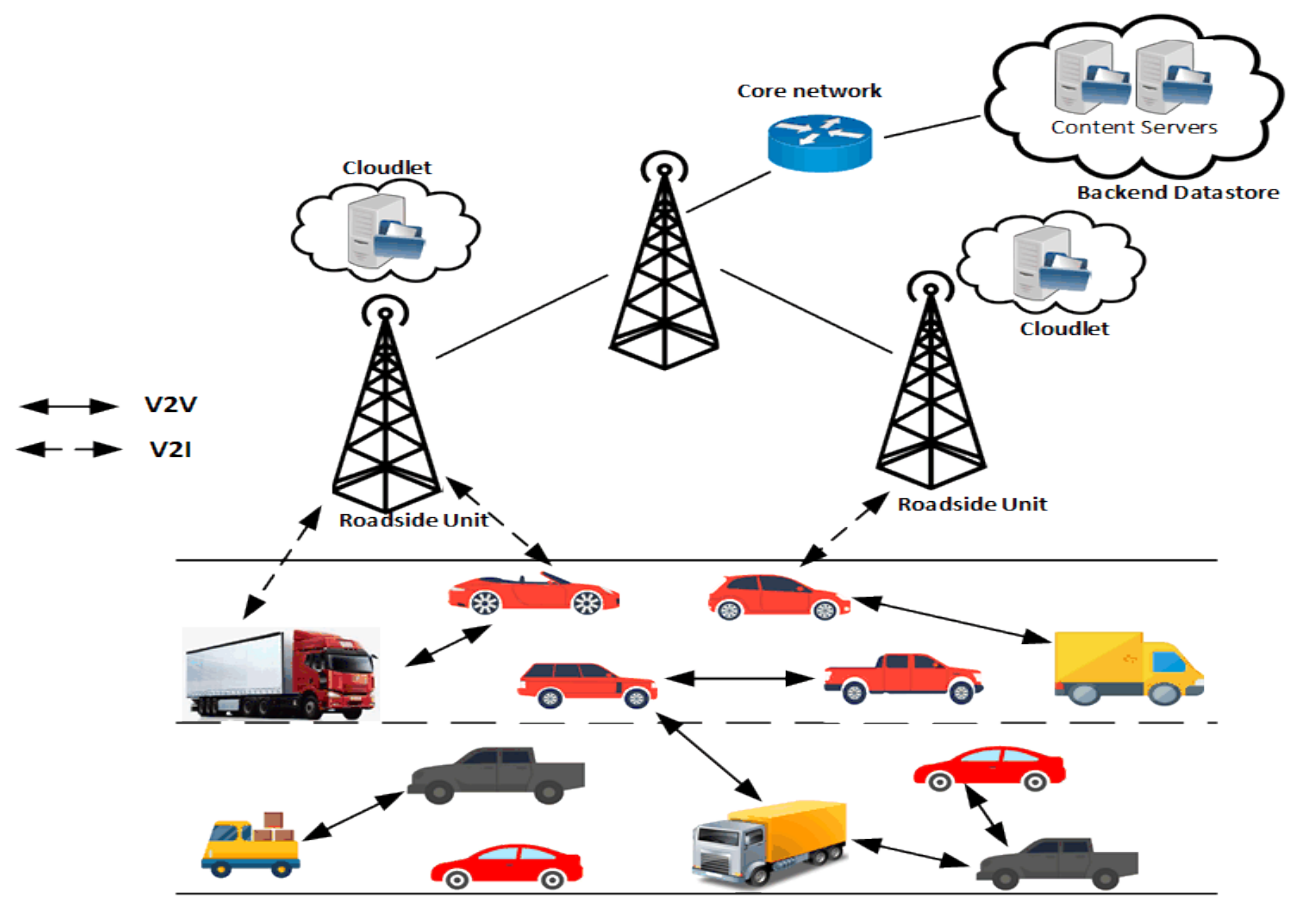

Figure 1: Tactile Network Inspired VANET

act as a router for its neighboring nodes, contributing to network formation and maintenance. Furthermore, such nodes are also envisioned to address the ad-hoc and dynamic nature of the network, taking into account node additions and failures. Mobile Adhoc Networks (MANETs) form the foundation layer of such infrastructures as these enable forming autonomous, decentralized networks without requiring prior installation of fixed and expensive communication infrastructures [11]. Consequently, MANETs are considered as a potential building block to achieve Tactile Internet as these can facilitate autonomous, decentralized communication among devices such as sensors and vehicles $[1,11]$.

Among other applications of MANETs, autonomous Vehicular Adhoc Networks (VANETs) is an emerging application of MANETs. Figure 1 represents an example system model for a connected vehicular network, which consists of two main participants: vehicles each with a wireless communication link and the Roadside Units (RSUs). The communication between vehicles is termed as Vehicleto-Vehicle $(\mathrm{V} 2 \mathrm{~V})$ communication and the communication between vehicles and RSU is termed as the Vehicle-toInfrastructure (V2I) communication. The mobility patterns of vehicles within this communication model can affect the quality of data exchange between devices in a tactile network.

Several mobility models exist which describe mobility patterns of mobile users in MANETs. These networks have diverse applications including content dissemination, content discovery and resource sharing among connected devices. MANETs are similar to P2P networks with respect to content discovery and dissemination [12, 13, 14]. Both networks share many common characteristics such as; selforganizing, no central administration for topology control, and nodes can leave or join the network at any time without any permission. A mobility model defines the movement patterns of mobile nodes and is an important feature for which the performance of protocols should be evaluated. Thus, it is essential to study and analyze the effect of content discovery under various mobility models.

There are two categories of mobility modeling. The first category includes a mobility model that considers the real movement patterns of mobile nodes in a real-world scenario and a second category which includes analyzing the mobility models on the basis of random mobility models. The mobility models can influence the mobility behavior of mobile nodes and the operation of underlying protocols. The widely adopted mobility models are the Random WayPoint (RWP) mobility model, Reference Point Group (RPG) mobility model, and Manhattan Grid (MG) mobility model. They have been used in theoretical studies and analysis. The widely used content discovery protocols in P2P setup are Gnutella -- a flooding-based content searching technique [15], and Chord -- a distributed lookup protocol $[16,17]$.

Within this context, existing studies only focus on the content discovery in MANETs under RWP mobility model. The results obtained through RWP mobility cannot be compared with the other mobility models. Obviously, in a diverse mobility environment, the interference and fading affect the channel quality dynamically. Consequently, application efficiency is affected in such an environment. 
Although there is significant research with respect to $\mathrm{P} 2 \mathrm{P}$ protocols tailored to MANETs with RWP, there is a lack of knowledge about the impact of underlying mobility patterns on the performance of P2P protocol in MANETs.

In this paper, our objective is to evaluate the mobility patterns of mobile nodes in a MANET to evaluate the efficiency of $\mathrm{P} 2 \mathrm{P}$ application in Tactile Internet environment. To achieve this objective, we present a systematic framework to identify the effect of mobility patterns of mobile nodes on the quality of $\mathrm{P} 2 \mathrm{P}$ applications. The proposed framework considers direct and derived mobility metrics for the evaluation of mobility impact on a P2P content searching technique. The framework also analyzes the interplay between diverse mobility patterns and $\mathrm{P} 2 \mathrm{P}$ application metrics. We have considered Random Waypoint mobility model (RW), Manhattan mobility model, and Reference Point Group Mobility model (RPGM) as the mobility models and have selected Gnutella as the P2P content discovery for unstructured protocols. We validate the framework through a series of simulations in NS-3 simulator. The simulation results reveal that unstructured Gnutella protocol is resilient in some mobility scenarios with proactive routing and a diverse behavior.

Additionally, we focus on the impact of the different mobility models on the performance of P2P content discovery protocols. To evaluate this we adopted the mathematical model proposed in [18] to systematically analyze the impact of mobility on the performance of P2P content discovery protocols for MANETs in a broader Tactile Internet environment. Bai et al. [18] analyzed the impact of mobility model on the performance of routing protocols. However, along with routing protocols, we also consider performance of $\mathrm{P} 2 \mathrm{P}$ content discovery under different mobility models. A number of $\mathrm{P} 2 \mathrm{P}$ protocols have been evaluated over MANETs under Random Waypoint model and has been analyzed for energy consumptions, response time, and hit-rate [19]. In this paper, we consider key additional metrics such as ping (the transmission of the packet to the server) pong (the response from the server), search delay (response time) and throughput (data transmitted per second) along with additional mobility models. Major contributions of this paper are as follows:

- We systematically evaluate the performance of content discovery protocols for different mobility models in the MANETs considering three network characteristics i.e. transmission range, node mobility, and network size. We also analyzed the impact of network characteristics on the performance metrics such as hit-rate, ping pong, search delay, and throughput.

- We study and evaluate the content discovery for a number of mobility metrics that are linked duration, temporal and spatial dependence, node degree, and relative speed of the mobile node. To the best of our knowledge, this is the first study that collectively considers routing protocols and mobility metrics for
Tactile Internet in general and MANETs in particular.

Rest of the paper is organized as follows. Section 2 provides existing related and provides motivation for this work. Section 3 presents P2P protocol and mobility models. The mobility modeling metrics are discussed in Section 4 and simulation methodology is described in Section 5. Section 6 presents the outcomes of the experimentation including detailed analysis. Section 7 provides discussion on the simulation results highlighting a comparative analysis of the mobility models for different experimentation settings. Section 8 concludes the papers with some thoughts on future work.

\section{Related Work}

In this section, we present an overview of existing related work within the areas of Tactile networks, P2P, mobile ad-hoc networks, and mobility models.

Maier et al. [1] presented an overview of the advancements in Tactile Internet and identify challenges ahead. Of particular interest from this paper is a holistic architecture which presents an integrated vision of existing technologies such as Fog Computing, Mobile Edge Computing, 5G, Software Defined Networks, and Cloud robotics thereby identifying opportunities for further enhancements. Ateya et al. [20] introduced a structure for the Tactile Internet system making use of SDN and MEC at its core. The proposed system structure is composed of an end-user device, RAN (Radio access network), cloud servers, access points, data routers and switches, OpenFlow switches, Middleboxes and SDN (Software Defined Network) controller. The authors make use of SDN to reduce the latency with basic simulations that reveal delays of $0.95 \mathrm{~ms}$ and $1.22 \mathrm{~ms}$ for $1 \mathrm{Gpbs}$ and are expected to meet the $1 \mathrm{~ms}$ round-trip objective for Tactile Internet. Arjun et al. [21] proposed a design and implementation for an end-to-end Tactile Cyber-Physical System (TCPS) with the aim to demonstrate robotic execution of physical gestures/movements directed by a human and therefore focusing on haptic sensations. The experiments conducted by the authors featured capturing hand gestures made by a human using tactile gloves which are translated into the same action by a robotic arm. The authors have reported a round-trip time of $85 \mathrm{~ms}$ which is of course much larger than the expected $1 \mathrm{~ms}$ for Tactile networks however it signifies the need for enhanced communication technologies such as 5G.

Sreekrishna et al.[22] presented a prototype implementation of the Tactile network making use of mobile edge cloud to achieve low latency. The authors proposed using $5 \mathrm{G}$ along with intelligent application level migration techniques to achieve low latency which is of utmost importance in a gaming application. This work signifies the feasibility of mobile edge cloud to achieve minimal delays with experiments demonstrating a delay of $400 \mathrm{~ms}$ for remote gaming server which is reduced to approximately 
$1 \mathrm{~ms}$ when the gaming application is migration closer to the user. These experiments demonstrate the potential of using MEC and Fog computing to achieve the goal of $1 \mathrm{~ms}$ round-trip time set by the Tactile network.

Peer-to-peer network architectures support a variety of applications. These applications can be one of the following: communication and distributed computing ( $\mathrm{P} 2 \mathrm{P}$ telephony e.g. Skype), and content distribution (Napster, Gnutella). Content distribution systems are the most prominent type of $\mathrm{P} 2 \mathrm{P}$ applications, therefore; these applications can be used as an example for evaluating performance. Furthermore, content distribution protocols can be grouped on the basis of their structure: unstructured or structured. Examples of structured network include P2P infrastructures Chord [23], CAN [24], Tapestry [25], however popular unstructured network include Napster, Gnutella [26], [27], Kazaa [28] and Pastry [29]. In this paper, we mainly focus on an unstructured protocol i.e. Gnutella because of its popularity in P2P systems. In [30], Shah et al. presented a Gnutella like P2P network that achieves mobility requirement of the ad hoc network for optimized search performance. Simulations have been performed and results are presented for a number of metrics including message overhead and message response time. In [31], Woungang et al. analyzed the Chord lookup method in the mobile $\mathrm{P} 2 \mathrm{P}$ network that uses distributed hash tables to maintain and update the finger table. In the presence of mobility, system has been evaluated for the lookup delay time, hop count and network load.

Leonardo et al.[12],[32] studied the performance of unstructured P2P protocols running over MANETs for three routing protocols that are DSDV, DSR, and AODV and different network characteristics. They concluded that the routing protocols have adverse performance and that the performance of the protocol mainly depends on the operational environment of the protocol. Furthermore, Arshad et al. [33] performed a performance evaluation of SAODV to identify the overhead caused due to secure extensions. Fathy et al. [34] analyzed the mobility impact on content discovery techniques on a MANET by constructing a systematic framework by making some assumptions for geographical node distribution. Barjini et al. [35] presented an analytical model for comparing the performance of unstructured P2P content searching techniques but they only ranked the techniques based on application metrics without considering the impact of mobility patterns of the mobile node. The bandwidth consumption in an unstructured network can be decreased by using redundant query messages scheme which also increases the success ratio [36].

Gruber et al. [37] proposed an MPP (Mobile Peer-toPeer Protocol) to interlink MANETs' network layer and MANETs' physical layer. The model tries to reduce traffic overhead through location-aware services. Marques et al. [32] proposed a mechanism for reducing usage of network resources when compared with a traditional unstructured P2P system in MANETs. Content-driven routing and adaptive data dissemination algorithms were proposed to forward the queries intelligently in a peer-to-peer network which is built over MANETs [38]. In [39], a treeshaped structure called COMMN is introduced to tolerate the frequent topological reconfigurations and achieves high performance through repair strategies. Babaei et al. $[40,41]$ propose an adaptive method to optimize the random walk for content discovery. The authors analyzed its performance for two parameters i.e. hit-rate and response time which are derived from the content discovery protocol performance metric. They analyzed the impact of mobility with metrics such as hit-rate, response time and energy consumption. However, in this paper we are using additional performance metric i.e. application hops, routing hops and content search delay. Further, we also studied the affect of these metrics on the network size and mobility patterns.

\section{Content Discovery Protocols and Mobility Mod- els}

In this section, we discuss content discovery protocols used in the ad-hoc or P2P networks (structured and unstructured) and mobility models used in the evaluation i.e. Random WayPoint, Reference Point Group Mobility, and Manhattan Grid.

\subsection{Content Discovery Protocols}

Peer-to-peer (P2P) network does not assume any dedicated server for the communication; each node acts as a client and a server at the same time. Therefore, direct communication occurs between the peers. A content discovery system allows peers and nodes in the network to discover content published by other peers in the network. Several content discovery protocols have been proposed for the P2P networks. These schemes can be classified into two major categories: a structured content discovery, and an unstructured content discovery [42]. Our aim here is to provide enough background to understand the workings of structured and unstructured protocols. Further details can be found in [36] and [43].

\subsection{Mobility models in MANET}

As mentioned in [18], RWP is incapable of tracing enough mobility characteristics. To comprehensively evaluate the effect of mobility on content discovery technique, we choose the following mobility models that are expected to have a significant performance impact on P2P applications with varying mobility characteristics. To achieve our goal, we selected RWP, RPG, and MG based on their significant characteristics and applications in a real life scenario.

\subsubsection{Random WayPoint (RWP)}

Due to its ease of implementation, Random WayPoint mobility model is extensively used in the research community. In the original model which is based on [44], 


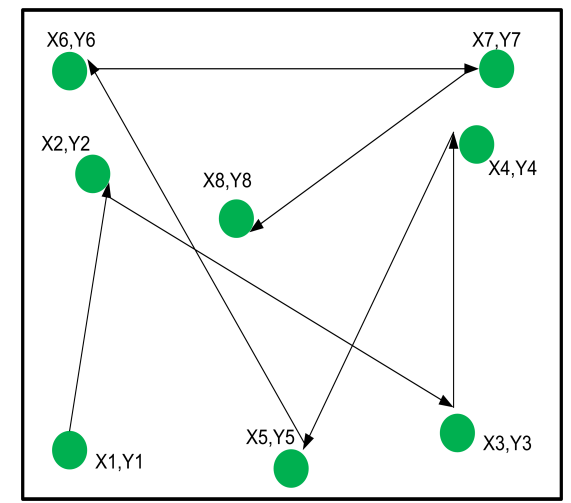

(a) Random Mobility

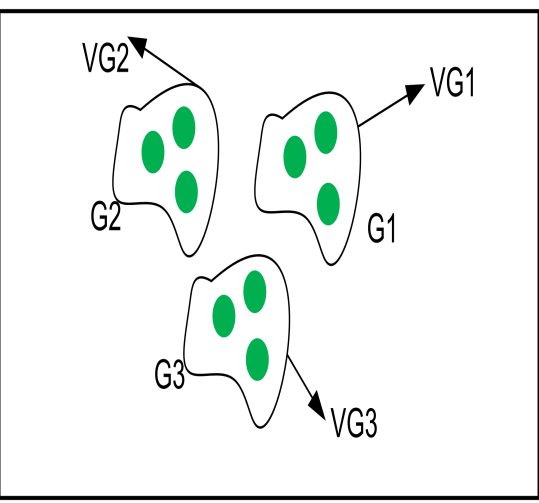

(b) Group Mobility

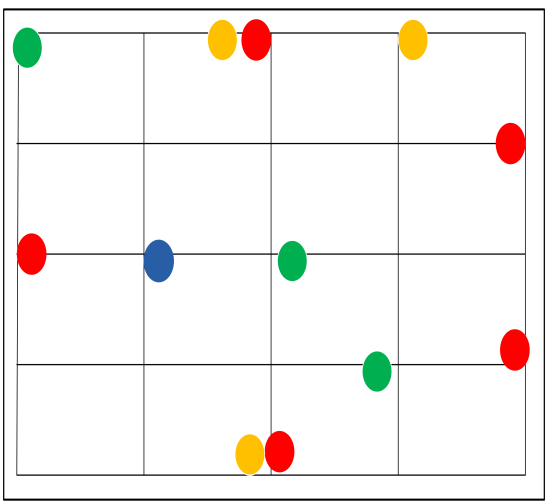

(c) Manhattan Mobility

Figure 2: Mobility Models in Mobile adhoc Networks : A) Random Mobility B) Group Mobility C) Manhattan

nodes are placed randomly in simulation region and in which each node continuously selects a random destination and moves toward it with a speed chosen from an interval [umin; umax]. When the node arrives at the destination, it waits for a specific pause time interval which is defined as a simulation parameter in [45], afterward the node selects a new destination and speed repeatedly until the end of simulation time. The downside of this model is that the environmental factors and non-environmental factors are not taken into account by the node.

\subsubsection{Reference Point Group Mobility (RPGM)}

In Reference Point Group Mobility, each group has a reference point (it is also called a logical center or group leader) and group motion behavior is realized by it [46]. In the beginning, each node is uniformly distributed around the reference point. The reference points change their absolute positions according to the arbitrary mobility model, but the relative positions inside a group they do not change. The application of RPGM widely used in the military battlefield where soldiers and commanders make a logical group for the common task.

\subsubsection{Manhattan Grid ( $M G)$}

Manhattan Grid model emulates the mobility pattern of streets and roads. This model uses maps for the movement patterns which are composed of horizontal vertical view of roads. In simulation grid area, nodes are randomly placed on the map where each node selects a direction and speed along the horizontal and vertical road on the map. As node reaches at the intersection of the horizontal and vertical road, it may change its direction (can turn right, left or go straight) with the certain probability. In this model, nodes only follow the predefined movements and speed and direction those are changed over time. Its application can be worthwhile in the modeling of an urban area where a ubiquitous computing service between movable devices is delivered and its characteristics are well described in [18].
The simplicity of the Random Waypoint model makes it the most widely used in MANETs simulation, however, MANETs can also be used in different applications where mobility of the user is quite different from the RWM. One such application of MANETs is military fields, movement in areas hit by disasters, and vehicular networks. Hence, there is a strong need to study the impact of $\mathrm{P} 2 \mathrm{P}$ content discovery protocols in MANETs under mobility models other than RWP. In these models, users usually move in groups and dependent on the history of their movements and their friends in the neighborhood. In this study, we choose RPGM and MG in addition to RWP because RPGM better characterize the mobility of users in military fields and MG characterizes the mobility of user in vehicular MANETs better.

\section{Modeling Mobility Models}

The quantitative and qualitative impact of different mobility patterns on $\mathrm{P} 2 \mathrm{P}$ protocol can be analyzed by considering several key mobility dependent and independent metrics [19],[47]. These metrics are defined as follows.

\subsection{Mobility Performance Metrics}

The direct mobility differentiates and measures the characteristics of dissimilar mobility patterns. These mobility patterns evaluate the characteristics of physical communication such as speed, acceleration, spatial dependence, temporal dependence, and geographic restrictions.

Relative Speed: Relative Speed is the speed between two objects in motion without refereeing any fixed point [18]. As tactile Internet requires delays to be less than $5 \mathrm{~ms}$, relative speed is critical to minimizing overall communication delays $[48,5]$. The relative speed between node $\mathrm{i}$ and $\mathrm{j}$ at time $\mathrm{t}$ can be computed as :

$$
R S(i, j, t)=\left|\vec{v}_{i}(t)-\vec{v}_{j}(t)\right|
$$

Then, the metric average relative speed $(\overline{R S})$ of all nodes over the time period $t$ can be computed as following. 


$$
\overline{R S}(i, j, t)=\frac{\sum_{i=1}^{N} \sum_{j=1}^{N} \sum_{t=1}^{N} R S(i, j, t)}{N(N-1)^{t}}
$$

where $(i, j)$ is the number of a node pair, $N$ is the total number of nodes in the network and $t$ is the time.

Degree of Temporal Dependence: It is degree of changes of the mobile node actual movement with respect to their past movements that are too far apart. This is significant for tactile networks as mobility can result in increased delays thereby disrupting expected communication patterns. The speed of node in the mobile continuously changes over the time and normally depend on the on the speed in the previous time. For each node in the network, the degree of temporal dependence can be defined as following:

$$
D_{t e m p}\left(i, t, t^{\prime}\right)=R D\left(\vec{v}_{i}(t), \vec{v}_{i}\left(t^{\prime}\right)\right) * R S\left(\vec{v}_{i}(t), \vec{v}_{i}\left(t^{\prime}\right)\right)
$$

The $D_{\text {temp }}\left(i, t, t^{\prime}\right)$ is a measure of the similarity of the speed of two mobile nodes that are not distant apart. $R D$ is the relative direction of nodes. The value of $D_{t e m p}\left(i, t, t^{\prime}\right)$ is high when a mobile node travel approximately in the same direction with the same speed over a certain time stamp. On the other hand, the value decreases if the relative direction or speed ration decreases. As this situation does not occur too far apart, therefore, the following condition is added:

$$
\left|t-t^{\prime}\right|>c \Rightarrow D_{\text {temp }}\left(i, t, t^{\prime}\right)=0
$$

Where $c>0$ is a constant.

The average value of $D_{t e m p}\left(i, t, t^{\prime}\right)$ is the value of average speed of nodes in the network over the entire time period and is computed as follows:

$$
\bar{D}_{t e m p}=\frac{\sum_{i=1}^{N} \sum_{t=1}^{T} \sum_{t^{\prime}=1}^{T} D_{t e m p}\left(i, t, t^{\prime}\right)}{P}
$$

Where in Equation 5, the $P$ represents the number of tuples $\left(i, t, t^{\prime}\right)$ such that $D_{t e m p}\left(i, t, t^{\prime}\right) \neq 0$.It can be observed that if the present speed of the node is completely independent of its speed at the previous time period, then the value of $\bar{D}_{\text {temp }}$ is expected to have small value for the $D_{\text {temp }}\left(i, t, t^{\prime}\right.$. However, if the current speed is dependent on the speed at the previous time period then $D_{\text {temp }}\left(i, t, t^{\prime}\right.$ will have high value.

Degree of Spatial Dependence: The degree of spatial dependence is a measure of the degree of the association of a node velocity with other node in the network which is not very far. This can be used to estimate the spatial dependency dimension of mobility which is critical to achieving performance goals set by tactile network and is computed as follows.

$$
D_{\text {spatial }}(i, j, t)=R D\left(\vec{v}_{i}(t), \vec{v}_{j}(t)\right) * S R\left(\vec{v}_{i}(t), \vec{v}_{j}(t)\right)
$$

Where $R D$ is the relative distance and $R S$ is the relative speed of the nodes in the network. The value of $D_{\text {spatial }}(i, j, t)$ is high when mobile nodes $i$ and $j$ travel approximately in same direction with same speed over a certain time stamp. On the other hand, the value $D_{\text {spatial }}(i, j, t)$ decreases if the relative direction or speed ratio decreases. As prevailing on to $D_{\text {temp }}\left(i, t, t^{\prime}\right)$, therefore the following condition is added:

$$
D_{i, j}(t)>c_{1} * R \Rightarrow D_{\text {spatial }}(i, j, t)=0
$$

Where $c>0$ is a constant.

The average value of $D_{\text {spatial }}(i, j, t)$ is the value of average degree of spatial dependence of all mobile nodes pairs in the network, i.e.

$$
\bar{D}_{\text {spatial }}=\frac{\sum_{t=1}^{T} \sum_{i=1}^{N} \sum_{j=i+1}^{N} D_{\text {spatial }}(i, j, t)}{P}
$$

Where $P$ represents the number of tuples $(i, j, t)$ such that $D_{\text {spatial }}(i, j, t) \neq 0$.. It can be observed that if mobile nodes move independently of one another, then the average temporal dependence is expected to have a small value.

\subsection{Derived Mobility Metrics}

In view of performance of $\mathrm{P} 2 \mathrm{P}$ protocol being impaired by the dynamic network topology. Therefore, metrics of the connectivity graph are selected to analyze the mobility influence on $\mathrm{P} 2 \mathrm{P}$ protocol. These metrics/properties can correlate with protocol performance The brief description of connecting graph metrics can be found in [49].

Number of Link Changes: Number of link changes is the number of times link shifts between nodes pair $i$ and $j$ over certain time interval. i.e.

$$
L C(i, j)=\sum_{t=1}^{T} C(i, j, t)
$$

Where $C(i, j, t)$ is an indicator random variable such that $C(i, j, t)=1 \Leftrightarrow X(i, j, t-1)=0$ and $X(i, j, t)=1$ i.e. if the link between nodes $i$ and $j$ is down at time $t$-1, but comes up at time $t$. The average vale of $L C(i, j)$ over node pairs can be computed as following:

$$
\overline{L C}(i, j, t)=\frac{\sum_{i=1}^{N} \sum_{j=i+1}^{N} L C(i, j)}{P}
$$

Where $P$ is the number of pairs $i, j$ such that $X(i, j) \neq 0$.

Node Degree: The degree of node $i$ is the number of neighbors of node $i$, i.e., the number of direct links a nodes has with other nodes. A node with a degree $\mathrm{d}=0$ is isolated, and a node with the degree $\mathrm{d}=\mathrm{N}-1$ is the well connected node. The average degree of a node $i$ in the graph 
$\mathrm{G}$ of $\mathrm{N}$ nodes is computed as :

$$
D_{i}=\frac{1}{N} \sum_{i=1}^{N} t_{i j}
$$

$t_{i j}=1$ when there is a link from $\mathrm{i}$ to $\mathrm{j}$ and 0 otherwise.

Number of Network Partitions: Network partition metric indicates the network distribution over entire terrain. A value of 1 for this metric means whole network is connected at all times i.e. any node has a path to any other node, a value larger than 1 shows that is not the case.

\subsection{Protocol Performance Metrics}

In this paper, we evaluate the performance of Gnutella protocol using five performance metrics which are details as following.

hit-rate (HR): hit-rate is a major metric of P2P network protocols evaluation. It represents the fraction of the queries that are successfully resolved in the P2P network. Assume that the network has N peers, where each node degree is $d \leq 4$. Then, the hit-rate for each peer during the course of the simulation time is given as:

$$
T Q_{a}=T F_{Q}+T S_{Q}
$$

Where $T Q_{a}$ is the total number of nodes visited for the content, $T F_{Q}$ is the number of nodes providing negative answer to query and $T S_{Q}$ reply positively to the query.

$$
T F_{Q}=\sum_{i=1}^{T Q_{a}}\left(T Q_{a}-T S_{Q}\right)
$$

And

$$
T S_{Q}=\sum_{i=1}^{T Q_{a}}\left(T Q_{a}-T F_{Q}\right)
$$

Thus, the average hit-rate of a peer in the network can be calculated as:

$$
A H R=\frac{\sum_{n=0}^{N-1}\left(T S_{Q} \operatorname{div} T Q_{a}\right)}{N}
$$

The average hit-rate (AHR) will be used in analysis part because it represents the average hit-rate at the network level.

Application Hops (AH): The application hop metric represents the path length in the overlay network. This path length could be different from the physical networks path length. Because the query visits an average number of peers hop to reach the desired content while looking for it. The shorter metric length indicates the efficient performance of content discovery protocol. During the course of the simulation, application hop from each peer node upon successful messages is measured as:

$$
A H\left(n_{i}\right)=\frac{\sum_{i=1}^{T S_{Q}}\left(T P_{v}\right)}{T S_{Q}}
$$

In Equation 16, $\mathrm{AH}\left(n_{i}\right)$ shows average application hops for one node. $T P_{v}$ is the number of nodes providing successful reply and, $T S_{Q}$ is the total number of nodes visited. Likely, Equation 17, the average application hops at the network level can be computed as follows:

$$
A A H=\frac{\sum_{n=0}^{N-1}(A H)}{N}
$$

Routing Hops (RH): The routing hops performance metric shows the path length in physical network or the requested query visits the number of network layers hops while searching the desired content. The value of this metric is always is expected to be higher than the $\mathrm{AH}$ metric, because every node in the network could not be a peer node. As RH value increases, the TTL value is decreased. The routing hops for each successful query are measured as:

$$
R H\left(n_{i}\right)=\frac{\sum_{i=1}^{T S_{Q}}\left(T N_{v}\right)}{T S_{Q}}
$$

Where $T N_{V}$ is the total number nodes visited for the query. The average routing hops at the network level is shown as:

$$
A R H=\frac{\sum_{n=0}^{N-1}(R H)}{N}
$$

Response Time (RT): Response time refers to the amount of time which is taken to return the query results upon success. It includes the delay which is perceived by a user who is requesting some contents, including the time for making the content request query to the nodes in the network, finding the appropriate node that has the desired content and responding back the response to the user. Each type of request has its own minimal response time. However, to evaluate performance of the system, we calculate the average response time of whole successful queries.

$$
R T\left(n_{i}\right)=\frac{\sum_{i=1}^{T S_{Q}} I_{c}+R_{c}+\left(\sum_{k=1}^{T N_{v}} R T T+e\right)}{T S_{Q}}
$$

Where in Equation 20, RTT is the round trip delay incurs in each hop when query is forwarded to neighbors node. $I_{C}$ and $R_{c}$ denote the query initiator and replier computation, in addition to these, $e$ denotes the network delay due to 
routing and queuing. Based on Equation 20, we can calculate average $R T$ at the system level similar to Equation 19.

Total Sent Messages (TSM): Total sent messages include the messages sent during simulation time by the application (query, reply, ping, pong, etc.), routing messages, and MAC layer messages. The purpose of this metric is to present the whole network state and traffic load in a different scenario.

\section{Simulation Methodology}

In order to achieve an in-depth evaluation of the content discovery for ad-hoc tactile networks, we have performed simulation experiments using the NS-3 simulator. Although there are alternatives available such as OMNET ++ , NS2, and GloMoSiM. NS3 is an open source feature-rich and widely used network simulator tools by the research community. It offers support for diverse technologies, wellknown programming languages $(\mathrm{C}++$ and Python), realtime integration and has been identified to offer best performance efficiency [50]. The simulations used two-rayground radio model and Gnutella as the content discovery technique which has been on top of UDP stack since TCP is considered less efficient for this kind of scenario. Initially the simulation network consists of 50 nodes which are uniformly distributed in a $1000 \mathrm{~m} \times 1000 \mathrm{~m}$ grid area. However, the experimentation with different network sizes has been performed as detailed in section 6.1. We consider AODV and DSDV being reactive and proactive routing protocols for the network layer. Table 1 summarizes the most significant parameters used in this study. IEEE 802.11 b radio interface, which is based on $914 \mathrm{MHz}$ Lucent WaveLAN is installed on each node with two-ray-ground radio propagation model. At any given instance of simulation, $75 \%$ nodes are always online while the remaining nodes join the P2P network at some time and leave after an interval. Joining and leaving time follows a uniform distribution. There are 250 files in the whole network because each node provides five different files. Each node queries $10 \%$ of the files with query times following a uniform distribution. Nodes move according to random waypoint, reference point group mobility and Manhattan grid patterns with a uniformly selected pause time 0 to 5 sec and average speed are also uniformly selected from 2 to $3 \mathrm{~m} / \mathrm{s}$. All the mobility traces have been generated using BonnMotion mobility tool [45].

Regarding Gnutella parameters, here we assume each node has the entry of 5 neighbors in its neighbor's list and 100 application messages in message cache. The TTL for queries is set to 10 and PING message is sent after every 10-sec interval. Experimental methodology is followed as in [19] and [43] and simulations are performed for 10 times respectively with different seeds for random number generator. Results have been produced and shown with $95 \%$ confidence interval.
Table 1: Simulation Parameters

\begin{tabular}{||c|c|c||}
\hline Parameters & Range & Default Value \\
\hline \hline Simulation area & (fixed) & $1000 \mathrm{~m}^{2}$ \\
Network size & $25-100$ nodes & 50 nodes \\
Node speed & $1-12 \mathrm{~m} / \mathrm{sec}$ & $2 \mathrm{~m} / \mathrm{sec}$ \\
Number of files & $50-250$ & 250 \\
Online nodes & (fixed) & $50 \%$ \\
Node placement & (fixed) & Uniform distribution \\
Communication range & (fixed) & $50 \mathrm{~m}$ \\
MAC layer & (fixed) & IEEE $802.11 \mathrm{~b}$ \\
Query message size & (fixed) & 64 bytes \\
Simulation time & (fixed) & $300 \mathrm{sec}$ \\
Mobility models & (various) & RW, RPGM, MG \\
\hline
\end{tabular}

\section{Performance Evaluation and Analysis}

In order to evaluate the effectiveness of various mobility metrics on P2P protocol over MANETs, we measure, study, and analyze impact of following key factors: network size, node mobility, and transmission range. This section presents the results which we have obtained through the stated methodology along with a thorough analysis.

\subsection{Network Size}

First scenario involved analyzing performance of P2P protocol (Gnutella) for various mobility patterns with respect to number of nodes in a MANET. The performance of P2P degraded as the number of hop counts between source and destination increased. This is because the search query has to go through more nodes before locating the desired content. In this scenario, the number of nodes are increased step by step keeping the number of queries per node consistent.

Figure 3 and 4 illustrate the performance of different P2P structure metrics for different mobility models under various network sizes. Figure 3. A shows that hit-rate value for various network sizes. The hit-rate for MG, RWP, and RPGM increases when the network size increases from 25 to 50 and then decreases afterward when the network size becomes greater than 50 nodes. The initial increase is because of the availability of multiple paths for content search. In all mobility models/patterns, the number of queries solved increase at first and have a peak value $84 \%$ with reactive routing when the number of nodes is 50 as shown in 3.A. On the contrary, the average hit-rate with proactive routing is very stable recording measurements from $56 \%$ to $93 \%$ for all network sizes. As the network becomes saturated, hit-rate value starts to fall till $1 \%$ in reactive and $56 \%$ in proactive for all patterns. The reduction of hit-rate in reactive mode coincides with a rocketed increase of search delay (response time) as well as a sharp decrease in ping/pong ratio. Additionally, in reactive mode, search delay only increases in dense networks whereas ping ratio decreased as can be seen in Figures 5. A and B. Therefore, it is identified that the performance impairment is caused by network density. We believe, as 


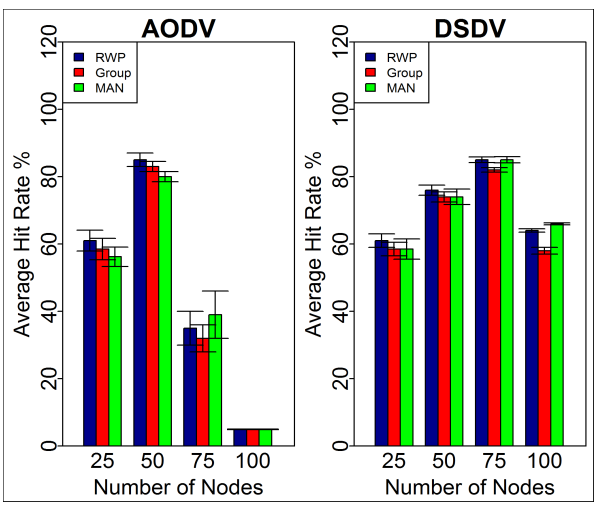

(a) Hit-Rate

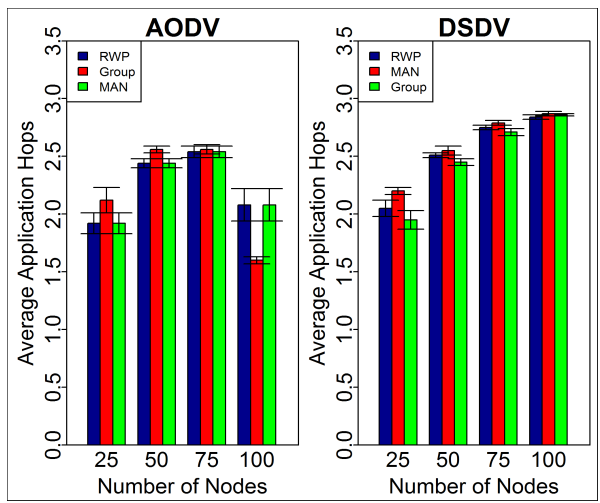

(b) Application-Hops

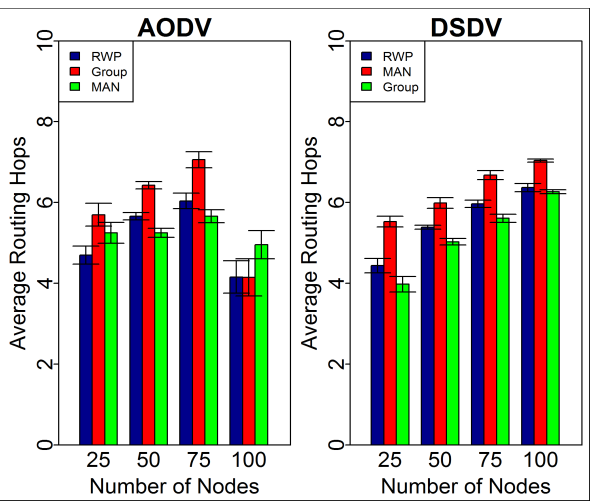

(c) Routing-Hops

Figure 3: Performance for the Network Size: A) hit-rate B) Application Hops C) Routing Hops

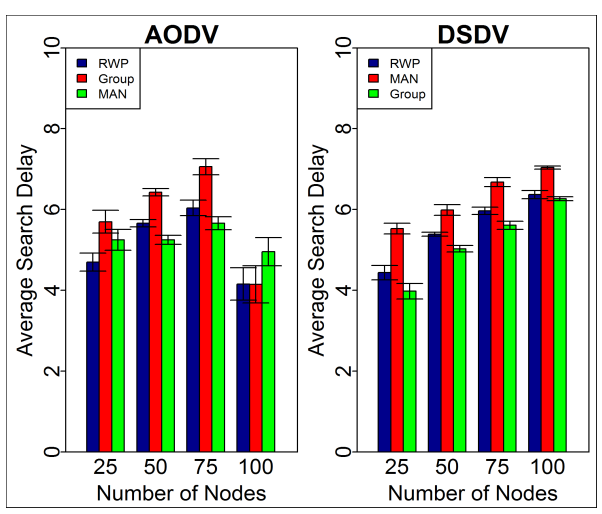

(a) Search Delay

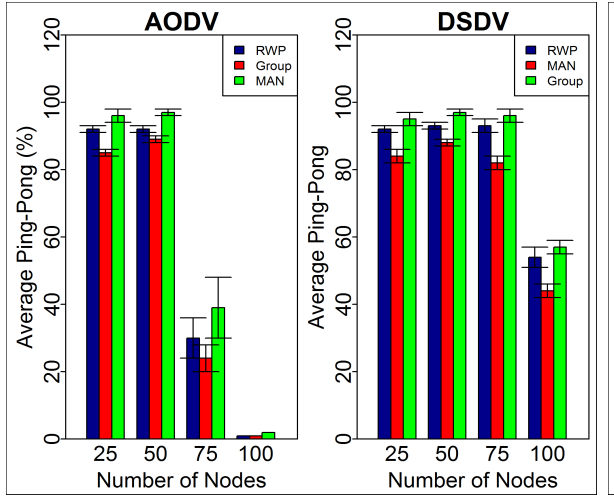

(b) Ping-Pong

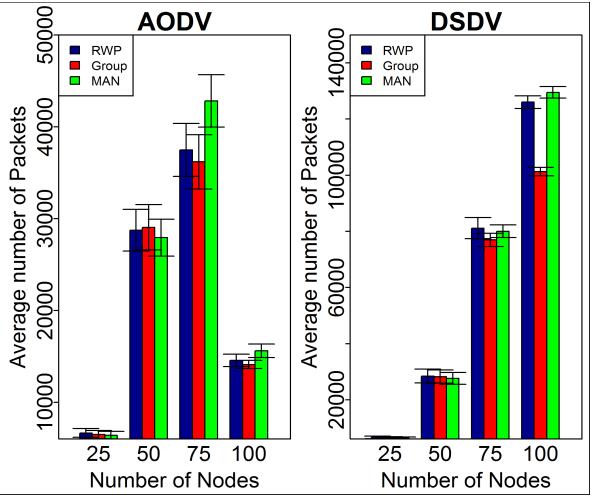

(c) Packets Sent

Figure 4: Performance for the Network Size: A) Search Delay B) Ping-Ping C) Packets Sent

the number of nodes increases in the same area, the wireless link gets more interference due to fixed transmission power. Thus, the more retransmissions occur at the MAC layer and routing control messages are produced dramatically.

For all mobility models, average application hop count for Gnutella is shown in Figure 3. B with network size. Initially, the protocol finds the desired contents within few hops whereas, in the proactive mode, the number of hops increase as the network size increases. However, the application hops with reactive routing tend to decrease when the network becomes dense. More importantly, Figure 3.C shows the hop counts at the routing layer against the number of nodes in the network. Similarly, Gnutella discovers the requested contents in physically sparse networks averagely within 6 hops for the reactive and proactive mode. When the network becomes dense enough (more than 75 nodes), Gnutella starts to locate the contents almost four hops away in reactive mode, while in the proactive mode it keeps increasing the number of routing hops with the network size. As we have already noticed, Gnutella has good performance with proactive routing in all the selected mobility models. From Figure 4.C, it can be seen that the average number of packets sent for the reactive protocol increases and then decreases as the number of nodes increases; however for proactive protocols, the number of packets sent is continuously increasing with the number of nodes in the network. This is because DSDV broadcasts every change in the network to every other node in a network and when using AODV, no such broadcasts occurs.

Figures 5 and 6 illustrate the effect of network size on direct and derived mobility metrics for various mobility models. RWP and RPGM have small but steady temporal dependency value in between -0.3 and .1 respectively. On the other hand, MG also has small dependence value but gets unstable as network size increases. This is because, nodes in the MG models keep moving alongside the streets, crossroads and randomly change their direction and destination at any time. Almost similar effect has been witnessed for average spatial dependence mobility metric but has positive dependence as shown in Figure 5.B. Spontaneously, the group leader in RPGM manages mobility of the mobile nodes and therefore this pattern has a higher spatial dependence. Furthermore, it leverages node grouping and distributes the network infrequently as compared with RWP and MG with higher network density as shown in Figure 5.C. Consequently, this connectivity problem is 


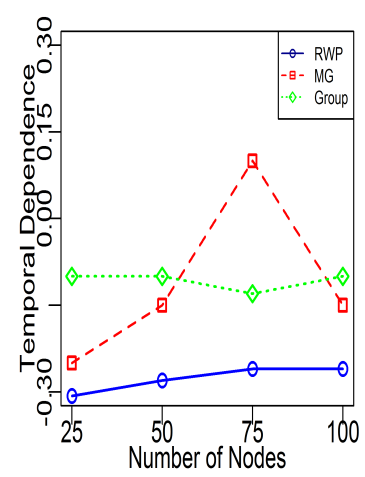

(a) Temporal Dependence

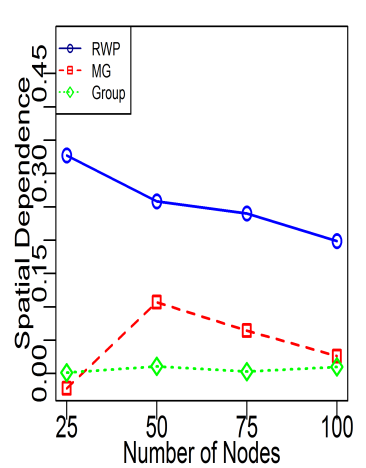

(b) Spatial Dependence

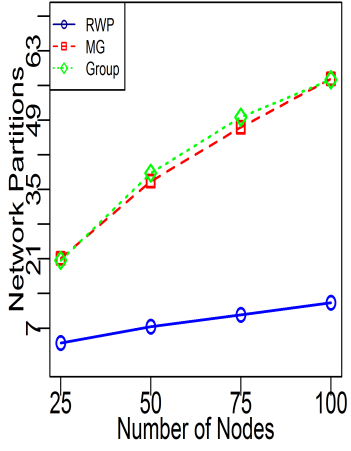

(c) Network Partition
Figure 5: Performance of Mobility Metrics for varying number of nodes: A) Temporal Dependence, B) Spatial Dependence and D) Network Partition

caused by the link impairment from other nodes interference.

The observation analysis from figure 6.A indicates that the relative speed ratio increases with the network size for all mobility models. Regarding Figure 6.B, the MG has lower average link duration than other two models but slightly similar to the RPGM. This is because MG has more movement pathways compared to RWP and RPGM and second nodes are always mobile. It is clear from Figure 6.B that an increase in network size has no effect on the link duration for MG and RPGM but link duration increases with increase in the number of nodes for RWP. The node degree for RWP model is larger than that of MG and RPGM due to majority of the nodes moving into the same direction with the passage of time. It can also be seen from Figure 6.C that network size has no major effect on the node degree of mobile nodes.

\subsection{Node Mobility}

Mobility plays an important role in wireless ad hoc networks and makes it distinct from the fixed network. Currently, P2P algorithms are being developed for wired networks where dynamics are much less common than in wireless ad hoc networks. Thus, we assess the impact of physical changes of the topology induced by frequent network partitioning on $\mathrm{P} 2 \mathrm{P}$ protocol for various mobility

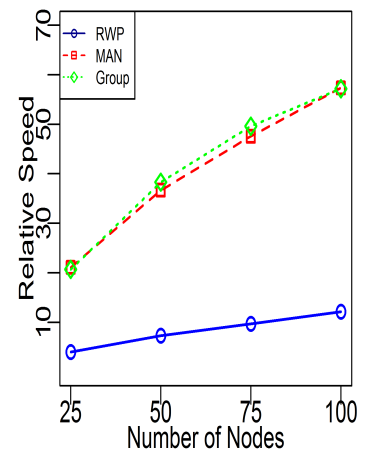

(a) Relative Speed

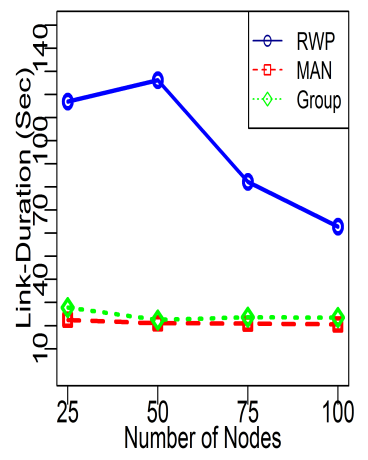

(b) Link Duration

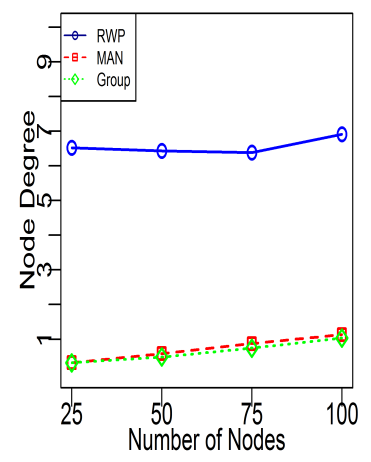

(c) Node Degree

Figure 6: Performance of Mobility Metrics For Network Size: A) Relative Speed B) Link Duration C) Node Degree

patterns. In this section, we study the Gnutella behavior by varying the average node speed from $0,2,4$, and 8 up to $12 \mathrm{~m} / \mathrm{s}$.

Figures 7 and 8 represent influence of mobility on Gnutella under various mobility models with reactive and proactive routing. For Gnutella P2P protocol, the increase in mobile speed does not have considerable effects on the hit-rate as shown in Figure 7.A. Surprisingly, the hit-rate for mobility models is $80 \%$ and almost remains constant in case of proactive mode even as the speed was increased as shown in Figure 7.A. This could be because a node with good connectivity can potentially discover contents quickly in its surrounding neighborhood. In case of reactive mode, it has a slight performance impairment with an increase in speed, especially for the Manhattan model. Protocol behavior can also be seen for ping/pong ratio as shown in Figure 8.B which represents the healthy state of the network. Both Manhattan and RWP models have a symmetric decreasing trend in both modes as the speed increases. Gnutella finds the requested contents almost within 2 to 3 application hops and averagely within six physically hops in all patterns and remains constant for all speeds for both routing modes as demonstrated by Figures 7 . A and B.

All mobility patterns have the same response time/search delay i.e. less than 1 second and remain under 1 second for 


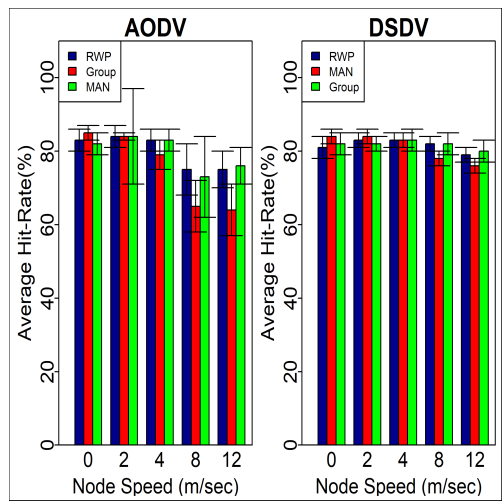

(a) Hit-Rate

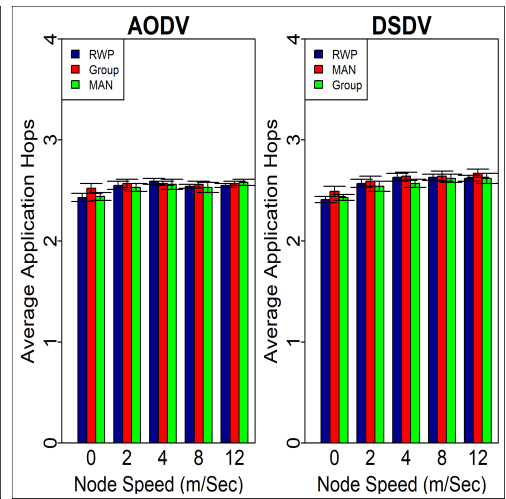

(b) Application-Hops

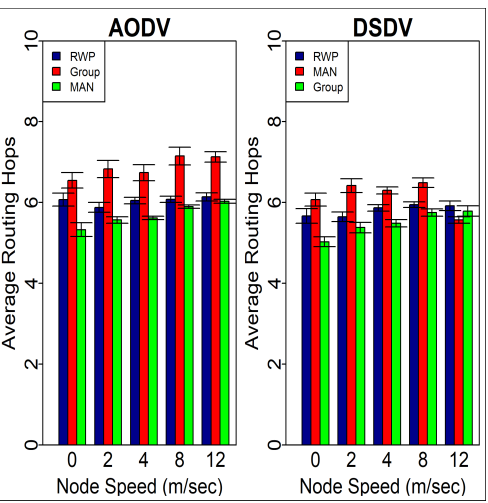

(c) Routing-Hops

Figure 7: Performance for Node Speed: A) hit-rate B) Application Hops C) Routing Hops

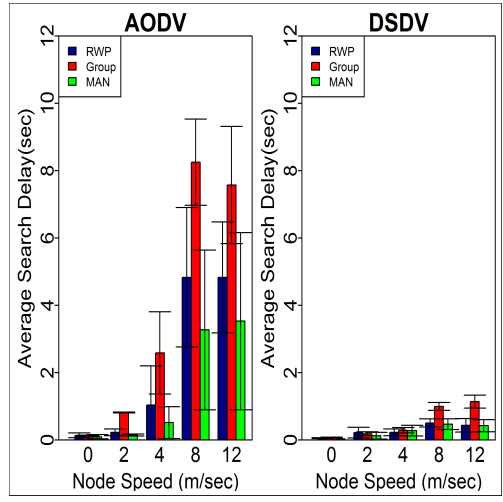

(a) Search Delay

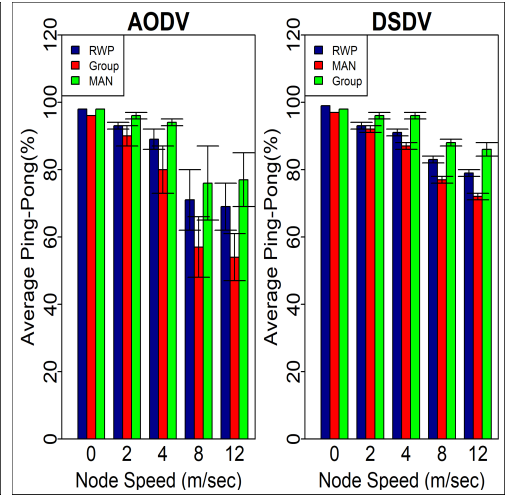

(b) Ping-Pong

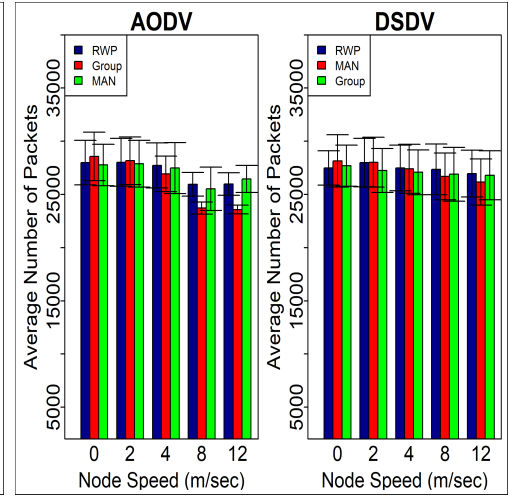

(c) Packets Sent

Figure 8: A) Performance for Node Speed: Search Delay B) Ping-Ping C) Packets Sent

various speeds as described in 8 . A expect for the reactive mode. In reactive mode, it has as increasing search delay fashion as the speed is increased. We believe it is caused by the strong influence of the underlying routing protocol. In the beginning, the messaging overhead slightly fluctuates with speed increments but later becomes stable for all mobility models. Due to clustering, RPGM has fewer or more messages overhead as compared to others models for the same performance. In general, we conclude that both routing modes with all mobility patterns have stable messaging overhead by varying node speed. The average number of packets sent for proactive and reactive protocol remain the same when the node speed changes but the number of packets sent in proactive protocols is greater than that of reactive routing as shown in Figure 8.C.

Figures 9 and 10 demonstrate the impact of various speeds on mobility metrics. As shown in Figure 9.A, RWP model has almost zero temporal dependence and remains stable as the speed of mobile nodes increases. The temporal dependence of RPGM and MG varies with the increase in mobile speed. RPGM has a higher spatial dependence around (0.4) as shown in Figure 9.B whereas both other models stay at around 0 even at high speeds. This is because, in RPGM, the group leader controls the movement of the node which results in high spatial dependence. On the other hand, nodes in RWP and Manhattan models select their own destinations regardless of others. As a result of grouping and coordination, RPGM also has fewer network partitions during the simulation course, as shown in Figure 9.C. Naturally, RWP and Manhattan have a similar trend to partitioning the network because of node individuality. As seen in Figure 10.A, relative speed has a lower value for RPGM and Manhattan about $(70,50)$, whereas for RWP has the highest and almost twice that for Manhattan. This is because the mobile nodes move in the opposite direction for both RWP and MG mobility models. Due to the factor of group movement, RPGM has high link duration and node degree values over both RWP and MG shown in Figures 10.B and C. The node degree of RWP first increases and then decreases as the mobile speed increases. However, the node degree for MG and RPGM first decreases and then remain constant even speed of mobile increases. All patterns have fully network connected in a static environment, but as speed is increased, nodes start to suffer significantly in RWP and Manhattan and could not establish a link for a long time. 


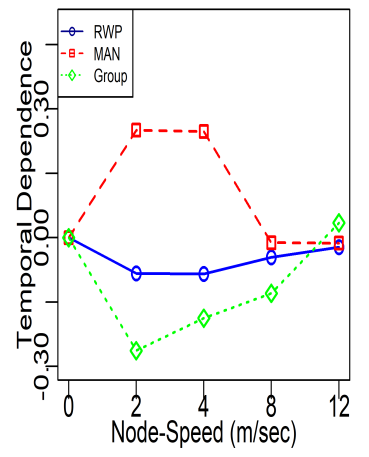

(a) Temporal Dependence

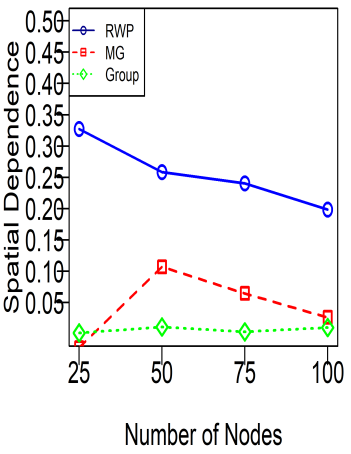

(b) Spatial Dependence

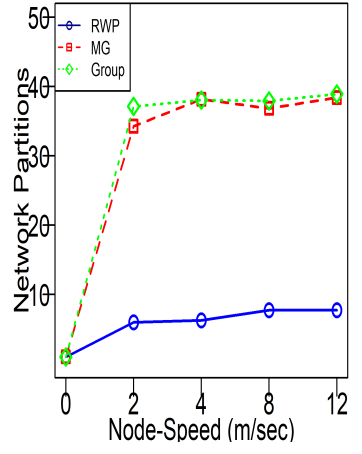

(c) Network Partition
Figure 9: Performance of Mobility Metrics For Node Speed: A) Temporal Dependence B) Spatial Dependence C) Network Partition

\subsection{Transmission Range}

It is important to evaluate the lower layers parameters influence on $\mathrm{P} 2 \mathrm{P}$ protocol. Therefore, the impact of transmission range on Gnutella that attains reasonable usage of energy in MANETs is studied regarding various mobility patterns. The transmission range is the range within which the mobile nodes receive signals from the other nodes and can extract meaningful information from that signal.

We experimented with different transmission power values to control the transmission range and its influence on Gnutella and mobility metrics. Experimental results are exhibited in Figures 11 and 12. The hit-rate is quite identical in both routing modes for all mobility patterns and has similar increasing or decreasing tendency when the transmission range varies. Overall, the Manhattan model achieves the worst hit-rate than the other two models. When transmission range stands at 20 meters it has $20 \%$ lower value in reactive compared to RWP and RPGM respectively, in Figure 11.A. However, as the transmission range is increased, the hit-rate unexpectedly improves in Manhattan, RWP, and RPG respectively.

A similar improvement is exhibited in Figure 12.B for ping/pong ratio with identical transmission range in all mobility patterns. Figure 11.B and C show transmission range with respect to application and routing hops high-

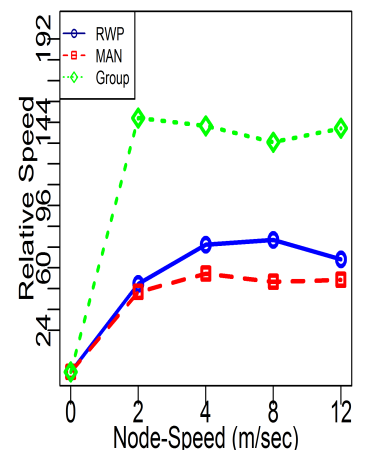

(a) Relative Speed

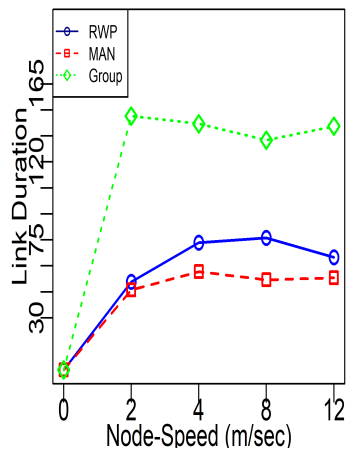

(b) Link Duration

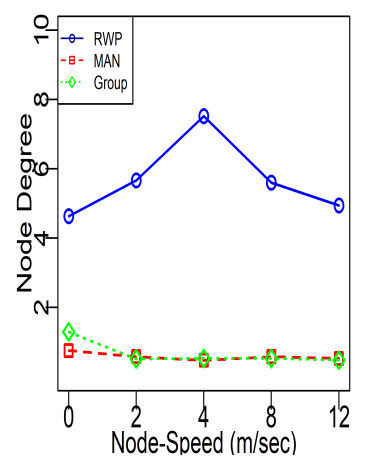

(c) Node Degree

Figure 10: Performance of Mobility Metrics For Node Speed: A) Relative Speed B) Link Duration C) Node Degree

lighting that by increasing the transmission range more number of peers are added in the neighbor's list. Consequently, application hops remain constant but routing hops decrease surprisingly. This can be because large transmission coverage area cover more nodes and has a higher probability to locate the contents in its vicinity. It also has an effect on search delay/response which becomes higher when the node covers a small area. On the other hand, when it starts to cover a large area and has interaction with other nodes, delay decreases even less than 1 second for both cases as presented in Figure 12.A. Furthermore, number of sent messages also verifies the importance of transmission range as presented in Figure 12.C. However, in limited transmission range, Gnutella has limited neighbors especially in Manhattan pattern with the reactive mode therefore very fewer messages are exchanged. Besides other transmission ranges, the same number of messages are exchanged in all mobility patterns with both routing modes.

Figures 13 and 14, show the impact of transmission range on mobility metrics for selected mobility patterns. In this section, the value for temporal dependence is not available as it does not correlated with transmission range. Therefore, temporal dependence is same for ranges in all mobility patterns. Figure 13.B highlights spatial depen- 


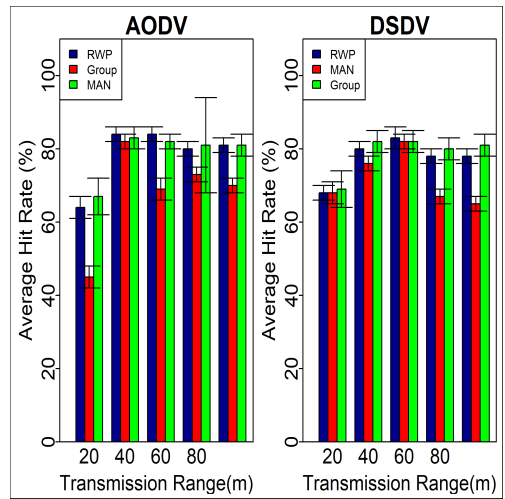

(a) Hit-Rate

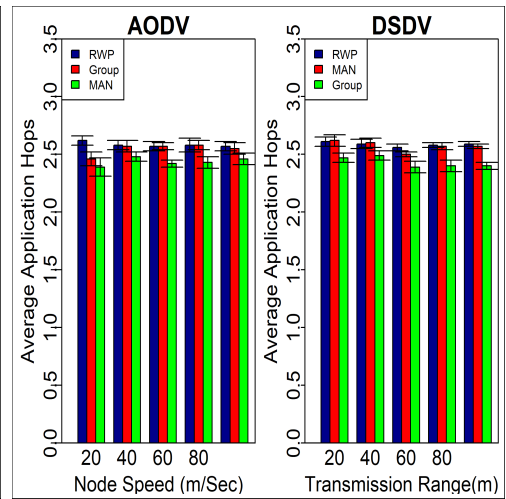

(b) Application Hops

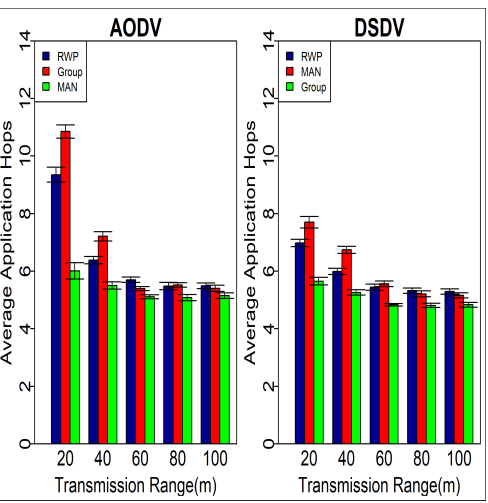

(c) Routing Hops

Figure 11: Performance for Transmission Range: A) hit-rate B) Application Hops C) Routing Hops

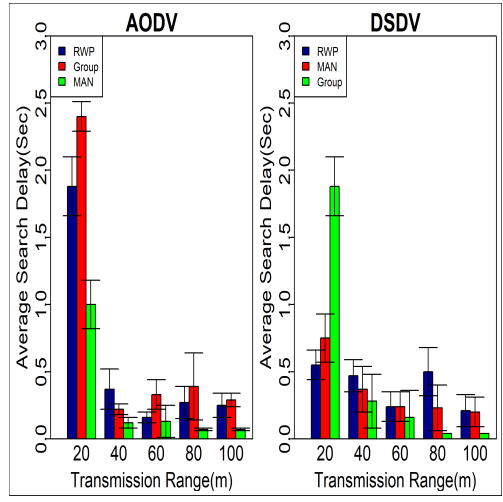

(a) Search Delay

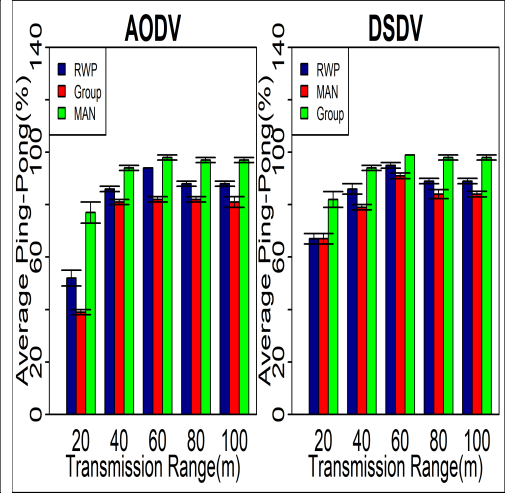

(b) Ping-Pong

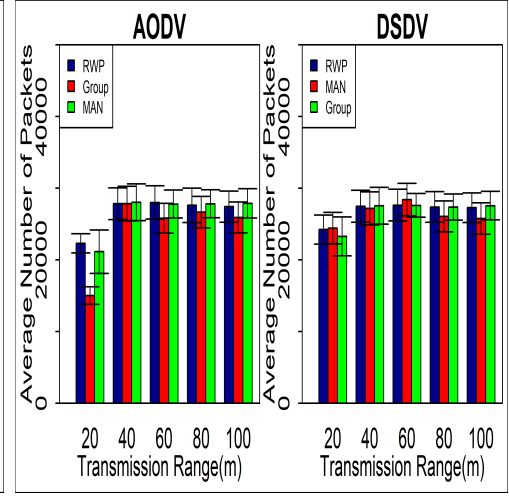

(c) Packets Sent

Figure 12: Performance for Transmission Range: A) Search Delay B) Ping-Ping C) Packets Sent

dence to have a larger value for RPGM i.e. almost six times more than RWP and Manhattan in small coverage area. With increase in transmission range, spatial dependence decreases gradually but still stays higher than others. As mentioned before, nodes in RPGM move in the form of swarm therefore with small transmission range they rely on each other. Figure 13.C illustrates the degree of network partitions highlighting that with few meters coverage the network scattered in several partitions even in RPGM. With good transmission range, the network starts to connect close enough for better communication. The same holds true for link duration and node degree in Figures 14 B and C. These elaborate that link duration lasts and number neighbors increase as coverage area is increased. The RPGM also suffers regarding relative speed by small transmission range, as shown in Figure 14.A. At the same time, RWP and Manhattan are not affected by transmission range.

\section{Discussion and Summary of Findings}

Experimentation involved evaluating the performance of different mobility models for the performance metrics in different settings. These settings included network size, transmission rate, and network mobility. This methodology provides rigorous experimentation to assess the effectiveness of mobility models to achieve performance goals of Tactile Internet. In this section, we summarize the results of experimentation as per the three settings i.e. network size, mobility, and transmission rate.

\subsection{Network size}

Our experiments have indicated that the hit-rate for MG, RWP, and RPGM increases when the network size increases from 25 to 50 decreasing afterward when the network size becomes greater than 50 nodes. We believe, the initial increase is due to the availability of multiple paths for content search. The average hit-rate with proactive routing is observed to be stable ranging from $56 \%$ to $93 \%$ for all network sizes. However, search delay in reactive mode increases in dense networks with ping ratio decreased as demonstrated by Figures 5. A and B indicating a performance reduction due to increased network density. For all mobility models, in the proactive mode the number of hops increases as the network size increases. However, the application hops with reactive routing tend to decrease when the network starts to get dense. Our evaluation has indicated that RWP and RPGM have low but steady temporal dependency value in between -0.3 and .1 respectively. 


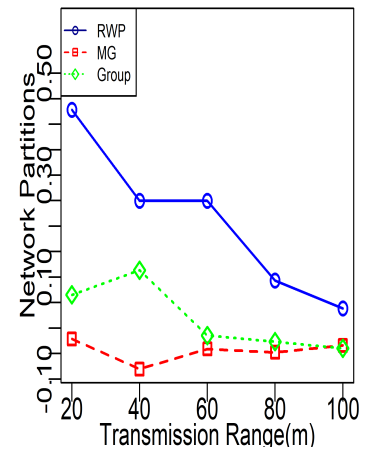

(a) Temporal Dependence

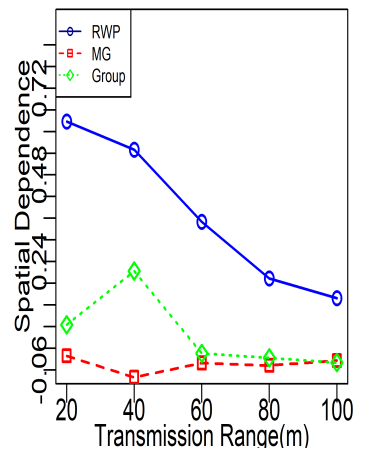

(b) Spatial Dependence

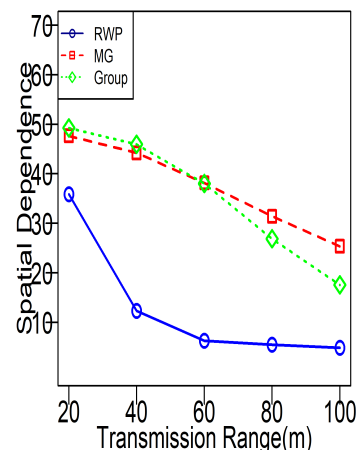

(c) Network Partition
Figure 13: Performance of Mobility Metrics For Transmission Range: A) Temporal Dependence B) Spatial Dependence C) Network Partition

However, MG also has low dependence value but it deteriorates with the increase in network size which can be due to the random change in the direction and destination of nodes in an MG model. Experimentation for spatial dependence evaluation identifies similar performance patterns for the mobility models. Furthermore, as indicated in Figure 6.A, the relative speed ratio increases with the network size for all mobility models. However, average link duration for MG is comparatively lower than other two models. We conclude that these results have a robust influence of low layers performance because due to the collision of data frames a packet has to wait in the queue and after a while, it might be discarded. Low layers behavior can be observed from each figure, as the number of nodes crosses more than 50, interference reaches a serious level. Consequently, we strongly affirm that the network density and proper topology has an effect on the P2P protocol.

\subsection{Node Mobility}

As demonstrated in Figures 7 and 8, the hit-rate is not influenced by an increase in mobility speed with it being $80 \%$ for all mobility models in proactive mode. However, the hit-rate deteriorates slightly with an increase in

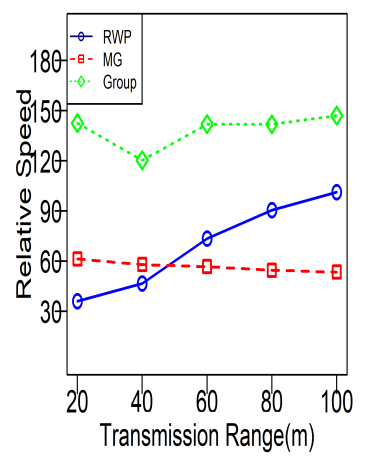

(a) Relative Speed

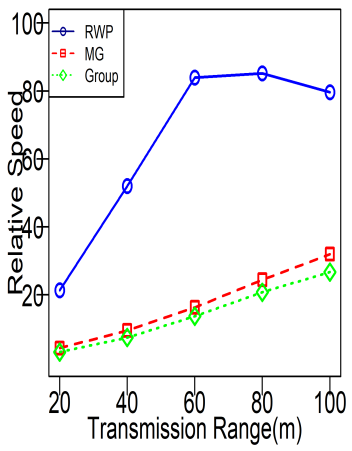

(b) Link Duration

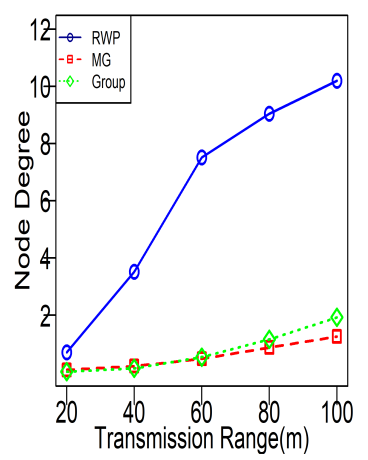

(c) Node Degree

Figure 14: Performance of Mobility Metrics For Transmission: A) Relative Speed B) Link Duration C) Node Degree

speed, especially for MG. Furthermore, all mobility patterns have similar response time/search delay, for different node speeds except for the reactive mode where it increases with an increase in the node speed. With respect to messaging overhead, an initial fluctuation is witnessed with an increase in speed which stabilizes for all mobility models. As is evident from Figures 9 and 10, the RWP model has minimal temporal dependence which is consistent across different node speed. However, the temporal dependence of RPGM and MG varies with the increase in node speed. Furthermore, RPGM demonstrated a higher spatial dependence whereas RWP and MG stay at around 0 even at high speeds. With respect to relative link speed, RPGM and Manhattan demonstrated lower measurements as compared to RWP which can be caused as mobile nodes move in the opposite direction for both RWP and MG mobility models. Additionally, due to group movement factor, RPGM has high link duration and node degree values compared to RWP and MG with the node degree of RWP reporting a varying trend for the increase in mobile speed. Our evaluation has clearly indicated that mobility has a significant influence on real-time applications but not much on delay tolerant applications such as P2P as we have witnessed the hit-rate to be satisfactory with all speeds. Therefore, all metrics exhibit the 
same performances with all velocities expect few fluctuations those are positively correlated with node speed and mobility metrics. We affirm it is due to the quick route changes/reconfigurations at the network layer.

\subsection{Transmission Range}

The experimentation performed as part of our evaluation encompasses different transmission power values to influence the transmission range and its impact on Gnutella and mobility metrics. As is evident in Figures 11 and 12 the hit-rate is identical in both routing modes for all mobility patterns and has similar increasing or decreasing tendency with varying transmission range. The experiments have identified MG to achieve worst hit-rate than the other two models however it improves with an increase in the transmission range, a trend which is also observed for ping/pong ratio for all mobility patterns. We have also analyzed the impact of transmission range on mobility metrics for selected mobility patterns. For instance, Temporal dependence is the same for all mobility ranges across diverse transmission ranges. However, for the smaller coverage area, spatial dependence demonstrates a high value for RPGM as compared with RWP and Manhattan which decreases gradually with the increase in transmission range. With respect to relative speed, RPGM is identified to be affected the most due to change in transmission range with minimal change for RWP and Manhattan. We conclude that transmission range/power plays a significant role in connecting the whole network in presence of running P2P applications. Since the success of this application depends on the number of available more users in the network. As reported above, Gnutella performance excels in RPGM due to its clustering nature, and mobility metrics become healthy when transmission range is good enough. Thus, it is certain that the transmission range parameter has a crucial role in the performance/evaluation of this application or network.

\section{Conclusions}

Tactile Internet evolves communications to encompass sensory information such as smell and haptic sensations combining ultra-low latency with extremely high availability, reliability, and security. Fog computing, Mobile Edge Clouds and MANETs have a profound role in its success. In this article, we studied and evaluated the impact of various key mobility patterns for unstructured content discovery techniques over MANETs in a broader Tactile Internet environment. The presented analysis and simulation results show that the Gnutella protocol discovers the contents in RPGM mobility pattern more efficiently than Manhattan and RWP patterns. Furthermore, we identified major performance impairments of Gnutella protocol in Manhattan pattern. Clearly, such impairments arise from the grid nature (with each grid area is $200 \mathrm{~m} 4$ ) of Manhattan yields the least connectivity time with peers nodes. Our simulation study shows that Gnutella protocol exhibits performance degradation and may not scale well for most mobility models in a highly saturated MANET environment with the increase of the nodes density and wireless channel dynamics.

Furthermore, we observe that the transmission power influences the network connectivity and mobility metrics. These influence Gnutella's performance consequently influencing the goal of Tactile Internet with respect to network delay and connectivity. To overcome such obvious limitations for the Gnutella protocol, we have made recommendations to improve the efficiency of the unstructured P2P protocols. The content discovery protocol should be designed to tolerate dynamics of the underlying network infrastructure taking into account characteristics of the wireless channels in Tactile Internet. Furthermore, content discovery protocol should be deployed with a cross-layer design which will provide protocol coordination with lower layers facilitating monitoring and adaptation to channel dynamics and network connectivity conditions.

\section{References}

[1] M. Maier, M. Chowdhury, B. P. Rimal, and D. P. Van. The tactile internet: vision, recent progress, and open challenges. IEEE Communications Magazine, 54(5):138-145, May 2016.

[2] M. Chen, Y. Qian, Y. Hao, Y. Li, and J. Song. Data-driven computing and caching in $5 \mathrm{~g}$ networks: Architecture and delay analysis. IEEE Wireless Communications, 25(1):70-75, February 2018.

[3] Yiming Miao, Yingying Jiang, Limei Peng, M. Shamim Hossain, and Ghulam Muhammad. Telesurgery robot based on $5 \mathrm{~g}$ tactile internet. Mobile Networks and Applications, Sep 2018.

[4] M. Simsek, A. Aijaz, M. Dohler, J. Sachs, and G. Fettweis. 5genabled tactile internet. IEEE Journal on Selected Areas in Communications, 34(3):460-473, March 2016.

[5] G. P. Fettweis. The tactile internet: Applications and challenges. IEEE Vehicular Technology Magazine, 9(1):64-70, March 2014.

[6] G. Fettweis and S. Alamouti. 5g: Personal mobile internet beyond what cellular did to telephony. IEEE Communications Magazine, 52(2):140-145, February 2014.

[7] T. S. Rappaport, S. Sun, R. Mayzus, H. Zhao, Y. Azar, K. Wang, G. N. Wong, J. K. Schulz, M. Samimi, and F. Gutierrez. Millimeter wave mobile communications for $5 \mathrm{~g}$ cellular: It will work! IEEE Access, 1:335-349, 2013.

[8] T. S. Rappaport, Y. Xing, G. R. MacCartney, A. F. Molisch, E. Mellios, and J. Zhang. Overview of millimeter wave communications for fifth-generation $(5 \mathrm{~g})$ wireless networks-with a focus on propagation models. IEEE Transactions on Antennas and Propagation, 65(12):6213-6230, Dec 2017.

[9] P. Porambage, J. Okwuibe, M. Liyanage, M. Ylianttila, and T. Taleb. Survey on multi-access edge computing for internet of things realization. IEEE Communications Surveys Tutorials, pages $1-1,2018$.

[10] Flavio Bonomi, Rodolfo Milito, Jiang Zhu, and Sateesh Addepalli. Fog computing and its role in the internet of things. In Proceedings of the First Edition of the MCC Workshop on Mobile Cloud Computing, MCC '12, pages 13-16, 2012.

[11] Niroshinie Fernando, Seng W. Loke, and Wenny Rahayu. Mobile cloud computing: A survey. Future Generation Computer Systems, 29(1):84 - 106, 2013.

[12] L. B. Oliveira, I. G. Siqueira, D. F. Macedo, J. M. Nogueira, and A. F. Loureiro. P2p over manets: Application and network layers routing assessment. In Mobile Peer-to-Peer Com- 
puting for Next Generation Distributed Environments. Information Science Reference, 2009.

[13] Bin Salem Ali and Chee Wan Tat. Survey of cross-layer designs for video transmission over wireless networks. IETE Technical Review, 29 (3),:229, 2012.

[14] Haseeb Zafar. A survey of qos-aware routing approaches for mobile ad hoc networks. IETE Technical Review, 29, May-Jun 2012.

[15] Yatin Chawathe, Sylvia Ratnasamy, Lee Breslau, Nick Lanham, and Scott Shenker. Making gnutella-like p2p systems scalable. In Proceedings of the 2003 Conference on Applications, Technologies, Architectures, and Protocols for Computer Communications, pages 407-418, 2003.

[16] Ion Stoica, Robert Morris, David Karger, M. Frans Kaashoek, and Hari Balakrishnan. Chord: A scalable peer-to-peer lookup service for internet applications. SIGCOMM Comput. Commun. Rev., 31(4):149-160, August 2001.

[17] Ion Stoica, Robert Morris, David Karger, M. Frans Kaashoek, and Hari Balakrishnan. Chord: A scalable peer-to-peer lookup service for internet applications. In Proceedings of the 2001 Conference on Applications, Technologies, Architectures, and Protocols for Computer Communications, pages 149-160, 2001.

[18] Fan Bai, Narayanan Sadagopan, and Ahmed Helmy. The important framework for analyzing the impact of mobility on performance of routing protocols for adhoc networks. Ad Hoc Networks, 1(4):383 - 403, 2003.

[19] L. B. Oliveira, I. G. Siqueira, D. F. Macedo, A. A. F. Loureiro, Hao Chi Wong, and J. M. Nogueira. Evaluation of peer-to-peer network content discovery techniques over mobile ad hoc networks. In Sixth IEEE International Symposium on a World of Wireless Mobile and Multimedia Networks, pages 51-56, June 2005.

[20] Abdelhamied A. Ateya, Ammar Muthanna, Irina Gudkova, Anastasia Vybornova, and Andrey Koucheryavy. Intelligent core network for tactile internet system. In Proceedings of the International Conference on Future Networks and Distributed Systems, pages 22:1-22:6, 2017.

[21] N Arjun, S M Ashwin, K. Polachan, T V Prabhakar, and C. Singh. An end to end tactile cyber physical system design. In 2018 4th International Workshop on Emerging Ideas and Trends in the Engineering of Cyber-Physical Systems (EITEC), pages 9-16, April 2018.

[22] S. Pandi, R. S. Schmoll, P. J. Braun, and F. H. P. Fitzek. Demonstration of mobile edge cloud for tactile internet using a 5g gaming application. In 2017 14th IEEE Annual Consumer Communications Networking Conference (CCNC), pages 607608, Jan 2017.

[23] Ion Stoica, Robert Morris, David Karger, M. Frans Kaashoek, and Hari Balakrishnan. Chord: A scalable peer-to-peer lookup service for internet applications. In Proceedings of the 2001 Conference on Applications, Technologies, Architectures, and Protocols for Computer Communications, SIGCOMM '01, pages 149-160, 2001.

[24] Sylvia Ratnasamy, Paul Francis, Mark Handley, Richard Karp, and Scott Shenker. A scalable content-addressable network. SIGCOMM Comput. Commun. Rev., 31(4):161-172, 2001.

[25] Ben Y. Zhao, John D. Kubiatowicz, and Anthony D. Joseph. Tapestry: An infrastructure for fault-tolerant wide-area location and. Technical report, Berkeley, CA, USA, 2001.

[26] The gnutella developer forum, gnutella protocol specification 0.6 .

[27] The gnutella developer forum, gnutella protocol specification 0.4 .

[28] Kazaa; multimedia file sharing progrm.

[29] Antony I. T. Rowstron and Peter Druschel. Pastry: Scalable, decentralized object location, and routing for large-scale peerto-peer systems. In Proceedings of the IFIP/ACM International Conference on Distributed Systems Platforms Heidelberg, Middleware '01, pages 329-350, 2001.

[30] Babar Shah and Ki-Il Kim. Towards enhanced searching architecture for unstructured peer-to-peer over mobile ad hoc net- works. Wireless Personal Communications, 77(2):1167-1189, 2014.

31] I. Woungang, F. H. Tseng, Y. H. Lin, L. D. Chou, H. C. Chao, and M. S. Obaidat. Mr-chord: Improved chord lookup performance in structured mobile $\mathrm{p} 2 \mathrm{p}$ networks. IEEE Systems Journal, 9(3):743-751, Sept 2015.

[32] A. Marques, F. Mira da Silva, and R. Rocha. P2p over mobile ad-hoc networks. In 2009 6th IEEE Annual Communications Society Conference on Sensor, Mesh and Ad Hoc Communications and Networks Workshops, pages 1-3, June 2009.

[33] J. Arshad and M. A. Azad. Performance evaluation of secure ondemand routing protocols for mobile ad-hoc networks. In 2006 3rd Annual IEEE Communications Society on Sensor and Ad Hoc Communications and Networks, volume 3, pages 971-975, Sept 2006.

[34] Mahmood Fathy, Kaamran Rahemifar, Hamideh Babaei, Morteza romoozi, and Reza Berangy. Impact of mobility on performance of $\mathrm{p} 2 \mathrm{p}$ content discovery protocols over manet. Procedia Computer Science, 10:642 - 649, 2012. ANT 2012 and MobiWIS 2012

[35] H. Ibrahim H. Barjini, M. Othman and N.I. Udzir. Shortcoming, problems and analytical comparison for flooding-based search techniques in unstructured p2p networks. Peer-to-Peer Networking and Applications, pages pp.1-13, May 2012.

[36] Diego N. da Hora, Daniel F. Macedo, Leonardo B. Oliveira, Isabela G. Siqueira, Antonio A.F. Loureiro, José M. Nogueira, and Guy Pujolle. Enhancing peer-to-peer content discovery techniques over mobile ad hoc networks. Computer Communications, 32(13):1445 - 1459.

[37] R. Schollmeier, I. Gruber, and F. Niethammer. Protocol for peer-to-peer networking in mobile environments. In Proceedings. 12th International Conference on Computer Communications and Networks (IEEE Cat. No.03EX712), pages 121-127, Oct 2003.

[38] Thomas Repantis and Vana Kalogeraki. Data dissemination in mobile peer-to-peer networks. In Proceedings of the 6th International Conference on Mobile Data Management, MDM '05, pages 211-219, 2005.

[39] L. Mottola, G. Cugola, and G. P. Picco. A self-repairing tree topology enabling content-based routing in mobile ad hoc networks. IEEE Transactions on Mobile Computing, 7(8):946-960, Aug 2008.

[40] Hamideh Babaei, Mahmood Fathy, and Morteza Romoozi. Modeling and optimizing random walk content discovery protocol over mobile ad-hoc networks. Perform. Eval., 74:18-29, April 2014.

[41] Hamideh Babaei, Mahmood Fathy, Reza Berangi, and Morteza Romoozi. The impact of mobility models on the performance of p2p content discovery protocols over mobile ad hoc networks. Peer-to-Peer Networking and Applications, 7(1):66-85, 2014.

[42] Elena Meshkova, Janne Riihijärvi, Marina Petrova, and Petri Mähönen. A survey on resource discovery mechanisms, peerto-peer and service discovery frameworks. Comput. Netw., 52(11):2097-2128, August 2008.

[43] Leonardo B. Oliveira, Isabela G. Siqueira, and Antonio A.F. Loureiro. On the performance of ad hoc routing protocols under a peer-to-peer application. Journal of Parallel and Distributed Computing, 65(11):1337 - 1347, 2005.

[44] D. B. Johnson and D. A. Maltz. Handbook in Mobile Computing. Kluwer Academic Publishers, Alphen aan den Rijn, Netherlands, 1996. Edited by Imielinski and Korth,.

[45] Nils Aschenbruck, Raphael Ernst, Elmar Gerhards-Padilla, and Matthias Schwamborn. Bonnmotion: A mobility scenario generation and analysis tool. In Proceedings of the 3rd International ICST Conference on Simulation Tools and Techniques, pages 51:1-51:10, 2010.

[46] Xiaoyan Hong, Mario Gerla, Guangyu Pei, and Ching-Chuan Chiang. A group mobility model for ad hoc wireless networks. In Proceedings of the 2Nd ACM International Workshop on Modeling, Analysis and Simulation of Wireless and Mobile Systems, MSWiM '99, pages 53-60, 1999. 
[47] Qunwei Zheng, Xiaoyan Hong, and Sibabrata Ray. Recent advances in mobility modeling for mobile ad hoc network research. In Proceedings of the 42Nd Annual Southeast Regional Conference, pages 70-75, 2004.

[48] F. Dressler, F. Klingler, M. Segata, and R. Lo Cigno. Cooperative driving and the tactile internet. Proceedings of the IEEE, pages 1-11, 2018.

[49] Santosh Kmuar and S. c. Sharma. Evaluation of direct and derived mobility metrics of mobility models and its impact on reactive routing protocols. International Journal of Computer Applications, vol.48(21):pp 40-49, June 2012.

[50] Atta ur Rehman Khan, Sardar M. Bilal, and Mazliza Othman. A performance comparison of open source network simulators for wireless networks. In IEEE International Conference on Control System, Computing and Engineering, pages 34-38. IEEE, 2012. 